\title{
Optimal Allocation of Three Modes for the Intercontinental Transportations of Mass-Market Products
}

\author{
Katsuhisa Okita \\ United Parcel Service Company 3-3-16 Ishida, Minato-ku, Osaka 552-0006 JAPAN \\ Tel: +81-6-4395-6715, Email: k-okita2010@ares.eonet.ne.jp \\ Takashi Amemiya ${ }^{\dagger}$ \\ Department of Business Administration, Setsunan University, 17-8, Ikeda-Nakamachi, \\ Neyagawa-shi, Osaka 572-7508 JAPAN \\ Tel: +81-072-839-9409, Fax: +81-72-838-6609, Email: amemiya@kjo.setsunan.ac.jp
}

Received, March 11, 2010; Revised, September 14, 2010; Accepted, October 4, 2010

\begin{abstract}
There is a trend toward the world's manufacturing sites moving to East Asia. After manufacture, these products are transported to the advanced nations for their consumption demands. Among such advanced nations, the U.S.A. has the largest demand, and then Japan and European countries follow. It should be noted that the infrastructures of the Asian districts used for the production sites are rather limited, and the volume of products transported from these districts of Asia to the U.S.A is becoming tremendously large. This situation is causing very serious traffic problems. New products are required to be transported swiftly by air. Once the consumption and market demands are stable however, the products should be sent rather slowly, but in larger amounts. However, the airports of China are quite restricted in capacity, while the transportation volume is becoming large. As a result, transportation cost and the time required for transportation are increasing. Now, a third method is appearing. This is the so-called Sea and Air transportation. The cost and time in transit of this mode take mean positions between Air and Ocean services. At present there exists no well-thought-out strategy for how to allocate these three methods of transportation. This paper is an attempt to theoretically describe this mechanism and to discover the optimal way to allocate the three modes. For this purpose, several mathematical properties of value and cost of the products are defined, and on this basis a simulation of the transportation is established.
\end{abstract}

Keywords: Three Mode-transportation, Optimal Allocation, Intercontinental Transportation, Sea-and-air

\section{INTRODUCTION}

It is a well-known fact that many advanced nations have established a large number of production facilities in the Far East to obtain lower labor costs and transport their products to the many consuming countries. These consuming countries are usually developed countries and, therefore, the products are concentrated in a rather small number of hub ports for their transportation. Among these products, there appear many seasonal items, such as textile products and Xmas gifts. Every year such kinds of products appear seasonally and their movements assume similar styles. Also, for some products that are not seasonal ones, such as cellular phones, manufacturers produce new models rather cyclically, and as a result the- ir movements assume the same "seasonal" style. We can assume from experience that the cycle is usually 6 months long, although the cycle has no special meaning.

Generally, transportation of these products has particular characteristics. New products should be sent as rapidly as possible. For that reason, air transportation is usually used at this stage. However, the more products there are to be sent by air, the more difficult transportation becomes, both economically and in the volume transported. The location of the production is rather concentrated in a small area, and as a result, the airports that can be used are also limited.

After the first stage of transportation, a slower but larger-capacity mode of transportation is needed. That entails shipment by sea. Transportation by sea is slow

$\dagger$ : Corresponding Author 
but at the same time the cost of transportation is very low and the amount able to be sent at one time is extremely large. The difference between these two methods is significantly large and is still becoming larger. At present, a third method is making itself felt. This is sea and air transportation. This is a combinational method of transportation. First the products are sent by sea to some neighboring airports and then they are sent by air to their final destinations. The most popular route is the one through Japan. The cost of this new method is almost half and the time required is only about double that of direct air service. Moreover, because of the sluggish economy in Japan, obtaining air services is not difficult. And as a result, the obtainable transportation capacity is larger than that available by direct air services from China. However, introducing this method raises the difficult problem of how this method should be utilized, and moreover how much should be sent by this method.

This also poses an important economic problem: how to develop the infrastructures of airports in Japan. In this article we consider mainly the first problem. The variation in demand quantity determines export volume and thus the modes and timing of transportation. Hiraki et al. (2008) proposed a way of determining the adequate transportation routes and quantity to improve the total lead-time, the inventory quantity at the depot, etc. This, however, is in relation to "the international cooperative global complementary production system" (ICG CPS) and the optimal allocation of three modes for the international transportations is not covered.

Only a few research studies have appeared regarding this problem (Okita, 2007; Takeyasu and Okita, 2007). The optimal calculation of the allocation of these methods is being first considered in this report. This paper is structured as follows. In part 3 the background is shown. Section 4 is devoted to several assumptions and the calculation of the profit. Consideration of the obtained results is shown in section 5. An example is shown in section 6 , where the expected profits of several modes for a simple example are calculated and compared. The concluding remark is given in section 7 .

\section{MOTIVATION-INTRODUCTION OF SEA AND AIR MODE}

The motivation for this study is the present situation of world production areas and their transportation systems. As was presented in the previous section, over the past several years many developed counties have constructed a considerable number of firms in the FarEast, especially in China. Among the Chinese districts the district surrounding Shanghai is the most popular. The products of these plants are usually transported to the U.S.A. or other advanced countries, namely consumer countries. The volume of transportation to the U.S.A. is tremendously large and it is not an exaggeration to say that it constitutes the global transportation environment.

However, transportation of products to the U.S.A. is concentrated in very few transportation facilities. Most products are gathered into the Shanghai district and use is made of Shanghai Airport for air transportation or Shanghai terminal for shipment. Particularly the traffic conditions at the airport are becoming the largest problem. Consequently, air transportation is coming to require a lot of time and its cost is increasing.

Regarding this point, a third transportation method is attracting the world's attention. This is the mode called sea and air transportation. In this mode the products are first sent to Japan by sea and then transported by air to the U.S.A. The time required for this mode is about a week to ten days, and its cost is about half that of direct air transportation from China to the U.S.A. However, this method is not applied regularly, but usually only optionally. If it is carried out in an efficient way, its cheap cost would be evident. One of the most important reasons for this is that the ships used for transporting goods from China are, on the return trip, used for transportation from Japan to China. Moreover the air freight cost from Japan to the USA is comparatively low. Given these conditions, we may say that this mode of transportation is fairly promising. If this method can be proved to be efficient, usage of this mode would be more systematic. This would be profitable for Japan. That is the main motivation for this report

However, a transportation strategy for optimally using this approach has not been developed up to now. This is also a motivation for this report. It is expected that as the result of this new proposal, an approach to improving the infrastructure of the traffic systems of Japanese ports and airports can be derived.

\section{SYSTEM DESCRIPTION}

In this section several characteristics of the system are first presented and then on the basis of certain assumptions, the way to find the optimal transportation approach is studied.

\subsection{Characteristics of the System}

It should be noticed that that seasonal products should be transported to the consuming districts as fast as possible once they are produced. They are new products and their swift appearance gives them great competitive advantages. After this first period, the products are sent in large quantities to respond to the steady demands of the consuming districts, and therefore their value decreases. These are the first characteristics

Assumption I: The value of the product monotonically decreases as transportation time increases after the first appearance to the market, eventually approaching a fixed value. 
How rapidly the value decays and how high the first value is varies greatly depending on the characteristics of the products.

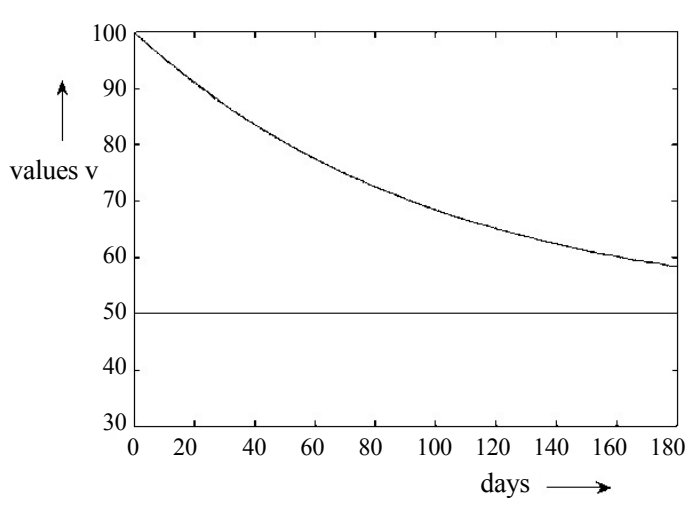

Figure 1. Example of value curve.

This is the basic assumption and there can be many variations depending on the products. The most important one is the situation of the consuming amount. It may change dynamically. For an advanced analysis, these situations should be considered.

At the same time, swift transportation is very expensive, and this is the second characteristic. Here the following assumption is introduced.

Assumption II: The transportation cost decreases monotonically as time passes. The cost by air is about a hundred times higher than that by sea. And the cost by sea and air is about half to one third of the cost by air.

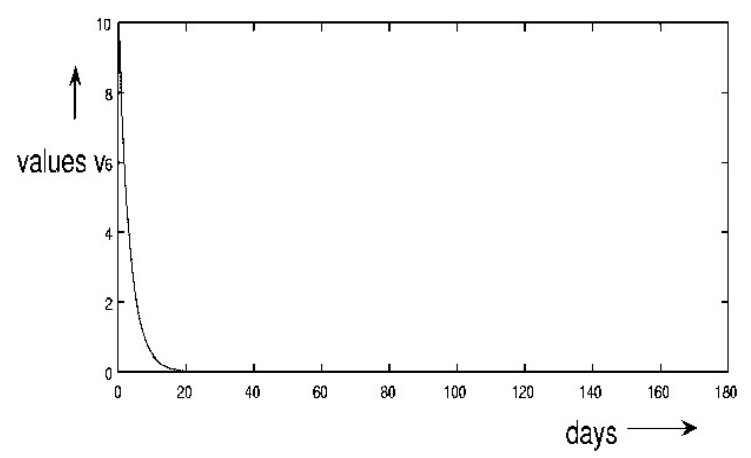

Figure 2. Example of transportation cost curve.

On top of the cost of transportation, the cost for the inventory should be considered. This is rather small compared to the transportation cost by air or by sea and air.

Assumption III: Inventory cost is a linear function of time and the amount of goods.

\subsection{Modeling and Optimal Unit Transportation}

For the analysis of this system several parameters and functions are necessary. For the sake of simplicity, transportation by air, by sea and air, by sea are called $\boldsymbol{A}$ mode, $\boldsymbol{S} \boldsymbol{A}$-mode, and $\boldsymbol{S}$-mode transportation respectively. Here the following parameters are introduced.

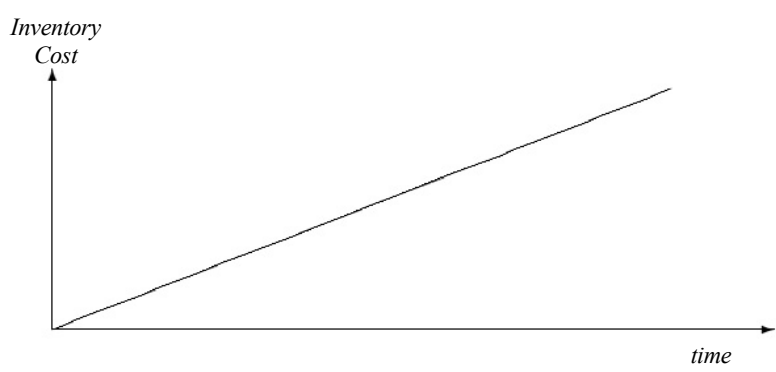

Figure 3. Inventory cost curve.

\subsubsection{Parameters}

$s$ : Amount of products sent at one time of transportation by $\boldsymbol{A}$-mode.

$\boldsymbol{d}$ : Time to produce the products of amount $\boldsymbol{s}$. Here, this is considered as a unit time.

$s$ : Amount of products that should be sent at one time of transportation by $\boldsymbol{S} \boldsymbol{A}$-mode.

$s$ : Amount of products that should be sent at one time of transportation by $\boldsymbol{S}$-mode.

$\boldsymbol{T}$ : Total production time.

(Note) The above parameters indicate that times of $s$ are sent at one time by $\boldsymbol{S} \boldsymbol{A}$-mode and times of $\boldsymbol{s}$ are sent at one time by $S$-mode. Usually $v>\mu$. These situations are caused by adopting containers for transportation by sea and not for air. For $\boldsymbol{S} \boldsymbol{A}$-mode, LCL (Less than Container Load) cargo is used.

\subsubsection{Functions}

On the basis of these assumptions, several nonlinear functions are needed for the mathematical analysis of the problem. First of all, value curve $f_{v}(t)$ and transportation cost curve $f_{t c}(t)$ must be assumed. They are defined as follows.

$f_{v}(t)$ : The value of one unit product at time $t$, which is produced at $\boldsymbol{t}=\boldsymbol{0}$. That means this function shows how rapidly the value of the product decreases.

$f_{t c}(t)$ : The cost of transportation of one unit product transported after $\boldsymbol{t}$ of its production.

$T_{i c}(t)$ : The cost of inventory of one unit for $t$ after its production.

Owing to the assumption of a monotone decrease in properties, we may choose an exponential function of time, such as

$$
f_{v}(t)=a e^{-\alpha t}+e
$$

as a candidate for the value curve, where $\boldsymbol{a}, \alpha, \boldsymbol{e}$ are constant, decided by the characteristics of the product. In 
the case of new models of a product, it is usually extremely high. That means $\boldsymbol{a}$ is rather large. This is also the case of a product in a severely competitive environment. Rapidly bringing it into the market is essential. The value decreases monotonically and it approaches a certain fixed value $\boldsymbol{e}$.

Also, as a candidate for the function for the transportation cost, the following function is given,

$$
f_{t c}(t)=b e^{-\beta t}+h
$$

where $\boldsymbol{b}, \quad \beta, \boldsymbol{h}$ are also constants, decided by the characteristics of the product, such as the size and other characteristics of the product. Of course, this curve does not mean there exists every service on this curve. It should be noted that this $\boldsymbol{f}_{t c}(\boldsymbol{t})$ indicates a cost of one time service to transport a unit product at $t$. It should also be noted that there exist only three services on this curve, that is $\boldsymbol{A}$-mode, $\boldsymbol{S} \boldsymbol{A}$-mode and $\boldsymbol{S}$-mode. In the usual case, the cost by air is about 100 times larger than that of by sea, and the time required for this transportation is 2 days for by air and 7 to 10 days for sea and air, and 20 to 30 days by sea. Comparison of the costs indicates that $\boldsymbol{h}$ is comparatively small and almost $1 / 100$ of $\boldsymbol{b}$, although it may not be zero. This situation implies that the cost curve approaches very near to the static value $\boldsymbol{h}$ after almost 30 days from the time the system started.

The inventory cost should be subtracted from these values. It is natural that the inventory cost is a linear function of time. Therefore, it is given as

$$
f_{i c}(t)=\gamma t
$$

Schematic views of these value functions and the cost function are given in Figure 1 and Figure 2.

Note that this is the cost of inventory for a unit product.

\subsubsection{Assumptions on the Modeling}

This report is almost the first trial of an optimal allocation of the transportation. Therefore, several assumptions for the modeling need to be introduced.

Assumption IV: Production speed is equal to the transportation ability by air. That means the production speed is $\boldsymbol{s} / \boldsymbol{d}$ throughout $\boldsymbol{T}$.

Assumption V: No lead time requirement exists.

As can be easily understood, this is a fairly restricted case and it is natural that there exists a demand from the destination region. It is also to be noted that the production speed is much higher than one day's production, and that the inventory amount increases as days pass. These cases are to be considered on the basis of this result. Even for the present restricted cases the necessity of three-mode transportation can be proven. It is shown in the following section.

\subsubsection{Calculation of Optimal Unit Transportation}

In this paragraph, as a basis of the theory for finding a total optimal strategy of transportation, the optimal method for only one unit of product is calculated first.

First, the profit which should be obtained by the transportation of a unit of product at time $t$ is calculated as follows.

$$
\begin{aligned}
f_{t p}(t) & =f_{v}(t)-f_{t c}(t)-f_{i c}(t) \\
& =a e^{-\alpha t}+e-b e^{-\beta t}-h-\gamma t
\end{aligned}
$$

The extreme point of the above function indicates the time at which the profit reaches the highest point

This point, if any, is obtained by calculating,

$$
\frac{\partial f_{t p}(t)}{\partial t}=-a \alpha e^{-\alpha t}+b \beta e^{-\beta t}-\gamma=0
$$

It is difficult to solve the above equation. The schematic view of the function is shown in Figure 4.

$$
f_{t p}(t)=f_{v}(t)-f_{t c}(t)
$$

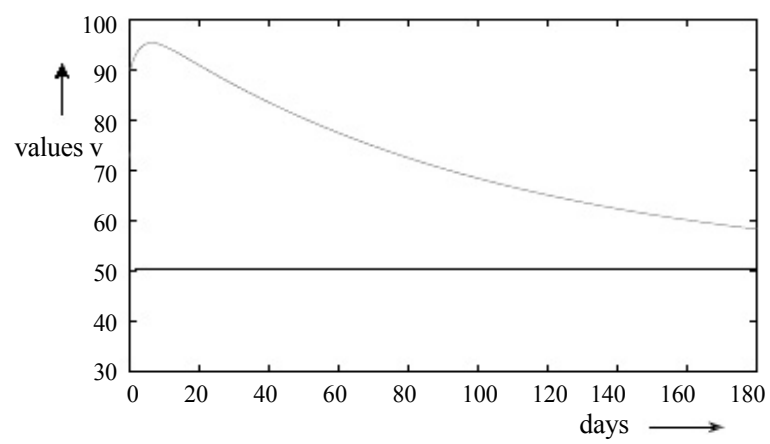

Figure 4. Value-Transportation Cost (0 days later).

It is found that there exists a desired point in this case. In this figure it is assumed that $a=10 b$ and $\beta=$ 30. It should be noted that this extreme point is for the product produced at $t=0$. Also note that the value curves change for the products produced later.

The value curve of the product arriving at the destination just after $d$ passed since the first product appeared, which is given as

$$
f_{d v}(t)=a e^{-\alpha(t+d)}+e
$$

Therefore, the total profit function for that product is now given as

$$
\begin{aligned}
f_{t p}(t) & =f_{d v}(t)-f_{t c}(t)-f_{i c}(t) \\
& =a e^{-\alpha(t+d)}+e-b e^{-\beta t}-h-\gamma t
\end{aligned}
$$

The views of these functions are given in Figure 4 
and Figure 5 for $d=0$ and $d=30$ respectively, both for $\gamma=0$. In these figures, the obtainable values, transportation costs subtracted, have peaks at 5 8days. That means the usage of the transportation requiring $5 \sim 8$ days are most efficient. It is clear that if $a \alpha \geq b \beta$ then there exists an extreme point for the products produced at $t=0$.

Figures so far considered are all for cases in which no inventory costs are included. We may assume that the inventory costs are comparatively low, since these products are transported rather soon after their production. However, in the case of $S$-mode it becomes comparatively large.

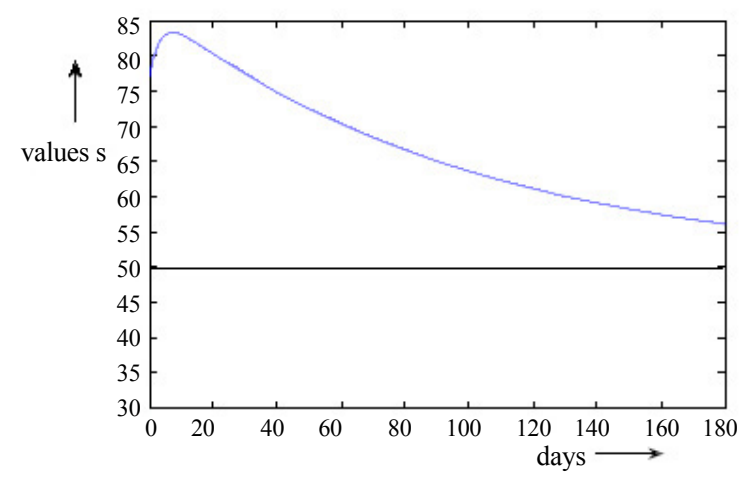

Figure 5. Value-Cost Curve (30 days later).

\subsection{Description of Problems}

To begin, in describing the problems the following restrictions must be introduced.

(H1) The production speed is constant throughout the entire production time.

(H2) Value curves and cost curve are fixed.

(H3) Inventory cost is a linear function of time.

(H4) The transportation should be restricted to three methods.

(H5) Each transportation method has a fixed transportation amount and until the amount of the products satisfy this restriction the transportation should not be performed, except the last one by sea.

(H6) The transportation amount by $S A$-mode is $\mu$-times larger than that by $A$-mode.

(H7) Transportation amount by $S$-mode is again $v$-times larger than that by $A$-mode.

(H8) Only one mode of transportation is selected at one time. This means the products should be transported by only one mode at each decision time.

(H9) Transportation by $S$-mode is not zero for all cases.

Reviewing these properties, the following considerations are derived.

Consideration 1: We may consider the transportation interval of A-mode consists of a time unit and may assume every decision is made at each unit time.
Consideration 2: At each time unit, the choice of the three modes is made

Consideration 3: By the properties of the monotone increasing characteristics of cost function and monotone decreasing value function, the decision times are divided into three periods, That is, the first period is assigned to A-mode and then to SA-mode, and the final period is to the $S$-mode of transportation.

As a result, the problem considered is:

Problem: Whether it is efficient to introduce the period of $S A$-mode? And if so, where are the points $t$ $=T_{S A}$ and $t=T_{S}$ which maximize the profit? Where $t=T_{S A}$ is the time at which $S A$-mode begins, $t=T_{S}$ is the time at which $S$-mode begins.

This situation indicates that $A$-mode transportation is performed from $t=0$ to $t=T_{S A}$. Then from $t=T_{S A}$ to $t=T_{S} S A$-mode transportation, and after $t=T_{S}$ only $S$ mode transportations are performed.

To mathematically analyze the problem, variables $x$ and $y$ are to be defined.

Definition:

$x$ denotes the number of transportations by $A$-mode; $y$ denotes the number of transportations by $S A$-mode.

Then the following relations hold.

$$
\begin{aligned}
& T_{S A}=x d \\
& T_{S}=x d+y d
\end{aligned}
$$
follows.

Then, mathematically the problem can be shown as

\section{Mathematical description of the problem}

Let the total profit obtained by the transportation using three modes be denoted as $T P_{\text {all }}(x, y)$ as a function of $x$ and $y$. Then the problem should be as follows.

Find $x$ and $y$ which satisfy

$$
\operatorname{Max} T P_{\text {all }}(x, y)
$$

Although this is the final objective, only the first part of the problem is considered here, because the problem becomes fairly complicated and is out of the range of this paper.

\section{CALCULATION OF THE PROFIT}

Here the total expected profit by the allocation of the three modes of transportation is calculated. It is assumed that $x$ times $A$-mode transportation and $y$ times $S A$-mode transportation are performed. It is also assumed that although all costs remain constant, the value 
function changes as time elapses

\subsection{Profit by $A$-mode Transportation}

By definition of unit time, at $t=d, \cdots, t=d x, A$ mode transportations are performed. Let $V_{\text {air }}$ denotes the total value obtained by one transportation by air, then the total value also depends on the amount to be sent, which is the same at each time by the assumption H5. Since the value of the products change as time $t$, the total value transported by air is given as

$$
V_{\text {air }}=\sum_{i=1}^{x} s f_{v(i-1) d}(d)
$$

Because

$$
f_{v(i-1) d}(d)=f_{v}(i d)
$$

we may write the above equation as

$$
V_{\text {air }}=\sum_{i=1}^{x} s f_{v}(i d)
$$

Let $T C_{\text {air }}$ denote the transportation cost by air, then it is given as

$$
T C_{\text {air }}=x s f_{t c}(d)
$$

As is assumed in H3, inventory cost is a linear function of time and the amount to be stored, and it is given as

$$
I C_{\text {air }}=\frac{1}{2} \gamma x d s
$$

The total profit by air is therefore given by

$$
T P_{a i r}=V_{a i r}-T C_{a i r}-I C_{a i r}
$$

Substituting (7), (8), (9) into (10), the following expression is obtained.

$$
T P_{a i r}=\sum_{i=1}^{x} s f_{v}(i d)-x s f_{t c}(d)-\frac{1}{2} \gamma x d s
$$

\subsection{Profit by $S A$-mode Transportation}

By assumption, the unit time for $S A$-mode is $\mu d$. After $x$ times $A$-mode transportations are performed, $S A$ mode transportations start. They are performed at $t=x d$ $+\mu d, t=x d+2 \mu d, \cdots, t=x d+y \mu d$. Following the previous subsection, the total revenue by $S A$-mode is calculated in this subsection. At each transporting time the value decreases as time $t$ increases. Since $y$ times of transportation are performed by this mode, the total value obtained by this transportation is given as,

$$
V_{\text {sea-air }}=\sum_{i=1}^{y} \mu s f_{v}(x d+i \mu d)
$$

For the calculation of the profit of the $S A$-mode the cost for this transportation should be subtracted from the calculated value in (12), as in the previous subsection. Therefore, the total profit for this mode is given as

$$
\begin{aligned}
T P_{\text {sea-air }}= & \sum_{i=1}^{y} \mu s f_{v}(x d+i \mu d)-y \mu s f_{t c}(\mu d) \\
& -\frac{1}{2} \gamma y(\mu d)(\mu s)
\end{aligned}
$$

\subsection{Total Profits of The Products}

After $A$-mode and $S A$-mode transportation, $S$-mode transportation begins. The starting times of this mode are $t=x d+y \mu d+v d, t=x d+y \mu d+2 v d, \cdots, t=x d$ $+y \mu d+r v d$, and $T$.

Following the previous subsection, the profit obtained by $S$-mode is calculated as follows:

First, the total value obtained by this mode is

$$
\begin{aligned}
V_{\text {sea }}= & \sum_{i=1}^{r} v s f_{v}(x d+y \mu d+i v d) \\
& +\frac{s}{d}(T-x d-y \mu d-r v d) f_{v}(T)
\end{aligned}
$$

where $r$ is defined as

$$
r=[(T-x d-y \mu d) /(v d)]
$$

and $[x]$ denotes the largest integer which does not exceed $x$.

Subtracting the total costs for this mode from the above value, the total profit is obtained as follows,

$$
\begin{aligned}
T P_{s e a} & =\sum_{i=1}^{r} v s f_{v}(x d+y \mu d+i v d) \\
& +\frac{s}{d}(T-x d-y \mu d-r v d) f_{v}(T) \\
& -r v s f_{t c}(v d)-\frac{1}{2} r \gamma(v d)(v s) \\
& -\frac{s}{d}(T-x d-y \mu d-r v d) f_{t c}(v d) \\
& -\frac{s}{2 d}(T-x d-y \mu d-r v d)^{2}
\end{aligned}
$$

where the second from the last term denotes the transportation cost of the remaining amount of the inventory transported at $T$ by sea, and the last term indicates the 
inventory cost of the same amount.

Overall profits obtained by these three modes of transportation are given as the summation of (11), (13) and (16)

$$
\begin{aligned}
T P_{a l l} & =\sum_{i=1}^{x} s f_{v}(i d)-x s f_{i c}(d)-\frac{1}{2} \lambda x d s \\
& +\sum_{i=1}^{y} \mu s f_{v}(x d+i \mu d)-y \mu s f_{t v}(\mu d) \\
& -\frac{1}{2} y \gamma(\mu d)(\mu s)+\sum_{i=1}^{r} v s f_{v}(x d+y \mu d+i v d) \\
& +\frac{s}{d}(T-x d-y \mu d-r v d) f_{v}(T) \\
& -r v s f_{t c}(v d)-\frac{1}{2} r \gamma(v d)(v s) \\
- & \frac{s}{d}(T-x d-y \mu d-r v d) f_{t c}(v d) \\
- & \frac{s}{2 d}(T-x d-y \mu d-r v d)^{2}
\end{aligned}
$$

This equation is the function of $x$ and $y$. Finding $x$ and $y$ to maximize the above equation is the problem to be solved.

\section{EXAMPLES}

\subsection{Assumptions and 5 Example Cases}

Finding the maximum value of the above equation on $x$ and $y$ is a rather tiresome problem, and moreover the variables $x$ and $y$ take only discrete values. Here a simpler illustrative example is given.

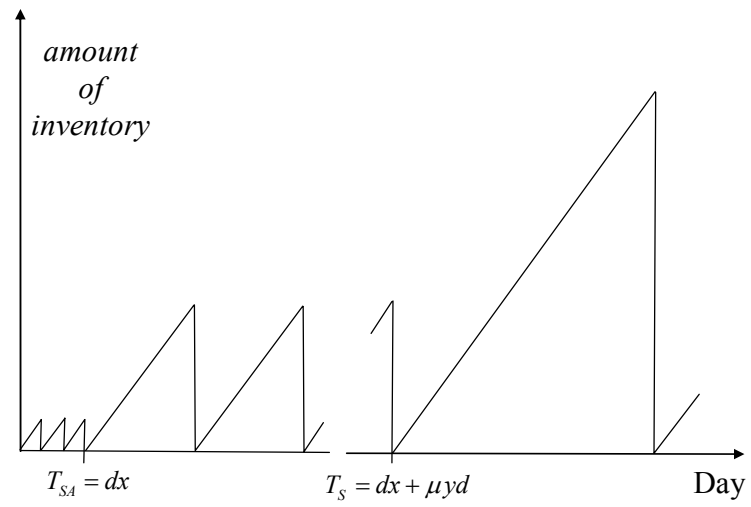

Figure 6. Transportation Strategy.

The value function and the cost function considered in this example are assumed to be simple linear functions, as follows:

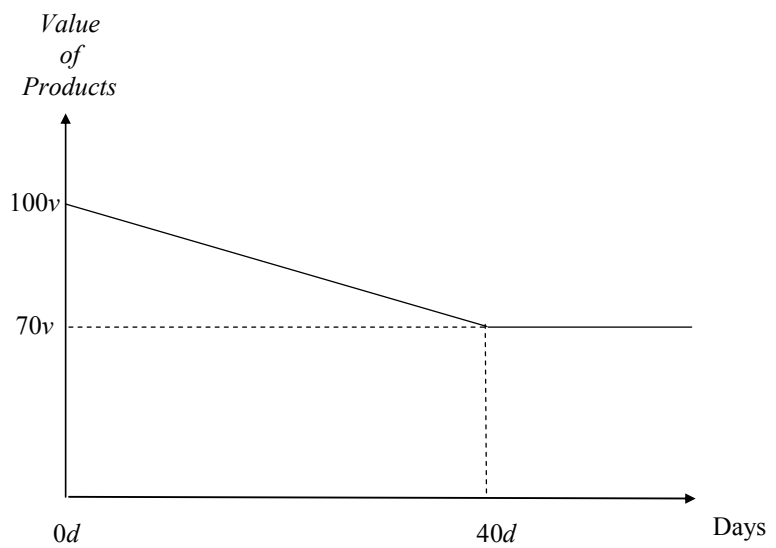

Figure 7. Value Curve of Example.

Here the parameters assumed are as follows:

1. $T=150 d$
2. $\mu=5$
3. $v=4$

To make the calculation easy for this problem, here it is assumed that the value curve and transportation cost curve are both linear, as in Figure 9 and Figure 10, respectively. Figure 9 indicates that at first the value of the product is $100 \mathrm{v}$ and it decreases linearly to $70 \mathrm{v}$ for $30 \mathrm{~d}$. After $30 d$ the value stays unchanged until $t=150 d$. The transportation cost curve of Figure 10 indicates that the transportation cost also decreases linearly for $20 \mathrm{~d}$. The transportation cost for $t=1 d$ is $10 v$. This is the cost for

$A$-mode. The cost for $S A$-mode is $5 v$ and the time required is $10 \mathrm{~d}$. The cost for $S$-mode is $0.1 v$ and the time required is $20 \mathrm{~d}$. After $20 \mathrm{~d}$ this cost also remains unchanged. For this problem 5 cases are considered.

(Case 1) (5 A-modes and $S$-mode)

In this case $A$-modes are performed 5 times and the remaining products are transported by $\mathrm{S}$-mode. Therefore, $T P_{\text {air }}$ and $T P_{\text {sea }}$ are given as

$$
\begin{aligned}
T P_{\text {air }} & =(99-10) v+(98-10) v+(97-10) v \\
& +(96-10) v+(95-10) v \\
& =435 v \\
T P_{\text {sea }} & =145(70-0.1) v=(10150-14.5) v \\
& =10135.5 v
\end{aligned}
$$

By $A$-mode transportation only one unit of product is transported at each time. The first $(\cdot)$ in the RHS of the first equation means the profit $99 v$ obtained by the first transportation by air costs $10 v$. The remaining terms are calculated as before. The remaining 145 units of products are transported by sea. The cost for the transportation by sea is only $0.1 v$ per unit. The total profit $T P_{\text {all }}$ in this case is given as 


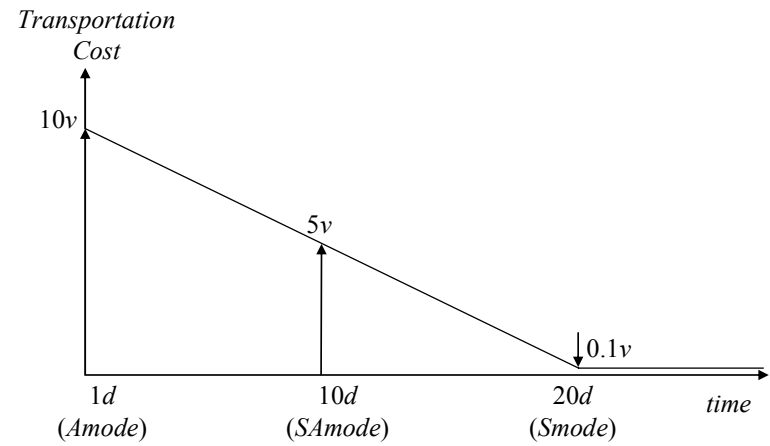

Figure 8. Transportation Cost Curve.

$$
\begin{aligned}
T P_{\text {all }} & =T P_{\text {air }}+T P_{\text {sea }}=435 v+10135.5 v \\
& =10135.5 v
\end{aligned}
$$

(Case 2) (5 A-modes $1 S A$-mode and $S$-mode)

In this case $A$-modes used 5 times, and $S A$-mode one time. The remaining products are transported by $S$-mode. Therefore, 5 units, 5 units and 140 units are transported by $A$-mode, $S A$-mode and $S$-mode, respectively.

The $T P_{\text {air }}, T P s_{\text {ea-air }}$ and $T P_{\text {sea }}$ are calculated as

$$
\begin{aligned}
T P_{\text {all }} & =(99-10) v+(98-10) v \\
& +(97-10) v+(96-10) v \\
& +(96-10) v=435 v \\
T P_{\text {sea-air }} & =5(80-5) v=375 v \\
T P_{\text {sea }} & =140(70-0.1) v=9800 v-14 v \\
& =9786 v
\end{aligned}
$$

The total profit $T P_{\text {all }}$ in this case is given as

$$
\begin{aligned}
T P_{\text {all }} & =T P_{\text {air }}+T P_{\text {sea }} \\
& =435 v+375 v+978 v=10590 v
\end{aligned}
$$

(Case 3) (5 $A$-modes $2 S A$-modes and $S$-mode)

In this case, $A$-mode is used 5 times and $S A$-mode is used 2 times. The remaining products are transported by $S$-mode. The $T P_{\text {air }}, T P_{\text {sea-air }}$ and $T P_{\text {sea }}$ are calculated as

$$
\begin{aligned}
T P_{\text {air }} & =(99-10) v+(98-10) v \\
& +(97-10) v+(96-10) v \\
& +(96-10) v=435 v \\
T P_{\text {sea-air }} & =5(80-5) v+5(75-5) v \\
& =375 v+350 v=725 v \\
T P_{\text {sea }} & =135(70-0.1) v=9450 v-13.5 v \\
& =9436.5 v
\end{aligned}
$$

The total profit $T P_{\text {all }}$ in this case is given as

$$
\begin{aligned}
T P_{\text {all }} & =T P_{\text {air }}+T P_{\text {sea-air }}+T P_{\text {sea }} \\
& =435 v+725 v+9436.5 v \\
& =10596.5 v
\end{aligned}
$$

(Case 4) $\}$ (4 $A$-modes $2 S A$-modes and $S$-mode)

In this case, $A$-mode is used 4 times and $S A$-mode is used 2 times. The remaining products are transported by $S$-mode.

The $T P_{\text {air }}, T P_{\text {sea-air }}$ and $T P_{\text {sea }}$ are calculated as

$$
\begin{aligned}
T P_{\text {air }} & =(99-10) v+(98-10) v \\
& +(97-10) v+(96-10) v=350 v \\
T P_{\text {sea-air }} & =5(81-5) v+5(76-5) v \\
& =380 v+355 v=735 v \\
T P_{\text {sea }} & =136(70-0.1) v=9520 v-13.6 \\
& =9506.4 v
\end{aligned}
$$

The total profit $T P_{\text {all }}$ in this case is given as

$$
\begin{aligned}
T P_{\text {all }} & =T P_{\text {air }}+T P_{\text {sea-air }}+T P_{\text {sea }} \\
& =350 v+735 v+9506.4 v \\
& =10591.4 \mathrm{v}
\end{aligned}
$$

(Case 5) (All $S$-mode)

As a comparison, here is a case in which all the products are sent by sea. In this case $T P_{\text {all }}=T P_{\text {sea }}$ and it is obtained as

$$
\begin{aligned}
T P_{\text {all }} & =T P_{\text {air }}=150(70-0.1) v \\
& =(10500-15) v=10485 v
\end{aligned}
$$

Summarizing the results, Table 1, as found below, is obtained., where $T C_{\text {all }}$ indicates the total of all transportation costs. In reference to Table 1 which consolidated respective performance on the 5 examples, although the standard number of transit-days is required for $S A$-mode, it varies depending on the routes and means of combination of the mode of transport. $S A$-mode gives flexibility of the transport getting the best $S$-mode and $A$-mode by being able to respond to the specific nature of the cargo, its condition, and the freight cost.

\subsection{Considerations on the Results}

In this example, the best result is obtained in Case 3 , meaning that the best result is obtained by introducing $2 S A$-modes and $5 A$-modes. At the same time, in this case the transportation cost is highest. It may be said that although the transportation cost becomes highest, the final gain is the largest in this case. It depends on whether it is more profitable to increase the number of $S A$-modes by decreasing the number of $A$-modes. In this example it is not more profitable. Although this example is a very simple one, we may safely suppose that the introduction of $S A$-mode transportation is profitable, and moreover, if it is possible to make this service a little more speedy, then this tendency will increase. This example is a very restricted one, such that the production speed is just the same as one day's transportation ability. 
Table 1. Obtainable value/cost comparison for 5 cases.

\begin{tabular}{|c|c|c|c|c|c|}
\hline modes & $\begin{array}{c}\text { 5A-modes } \\
\text { S-modes }\end{array}$ & $\begin{array}{l}\text { 5A-modes } \\
\text { 1SA-modes } \\
\text { S-modes }\end{array}$ & $\begin{array}{l}\text { 5A-modes } \\
\text { 2SA-modes } \\
\text { S-modes }\end{array}$ & $\begin{array}{l}\text { 4A-modes } \\
\text { 2SA-modes } \\
\text { S-modes }\end{array}$ & S-modes \\
\hline \hline$T P_{\text {air }}$ & $435 v$ & $435 v$ & $435 v$ & $350 v$ & 0 \\
$T P_{\text {sea-air }}$ & 0 & $375 v$ & $725 v$ & $735 v$ & 0 \\
$T P_{\text {sea }}$ & $10135.5 v$ & $978 v$ & $9436.5 v$ & $9506.4 v$ & $10485 v$ \\
\hline$T C_{\text {all }}$ & $64.5 v$ & $89 v$ & $113.5 v$ & $103.6 v$ & $15 v$ \\
\hline$T P_{\text {all }}$ & $10570.5 v$ & $10590 v$ & $10596.5 v$ & $10591.4 v$ & $10485 v$ \\
\hline
\end{tabular}

This is not the typical case and usually transportation begins after the amount of the product becomes sufficiently large. Optimal allocation of the transportation methods of such case is to be considered in the future.

\section{CONCLUSIONS}

It was shown that the importance and efficiency of the third method of intercontinental transportation of products are fairly large. This method is called sea and air because it uses these two modes. The most popular sea and air transportation is performed through Japan. It is rather a favorable situation for Japan, in terms of both sea and air transportation. A theoretical analysis for the optimal allocation of these three methods has been considered in this research for the first time.

Although the conditions or situations considered in this report are very primitive, the efficiency of this method has been explained theoretically. At present, this method is utilized quite optionally. It is desirable to carry out this method more systematically. In the application, the nonlinear functions for the value or cost functions must be estimated through theoretical and experimental considerations. These functions are much more complicated in reality and different for each product and the amount to be transported. Actually they should be constructed and polished for each product individually. Though this seems to be a fairly difficult task, it might be necessary in the future.

\section{REFERENCES}

Okita, Katsuhisa, Optimization in Intermodal Logistics (2007), Thesis for the Master Degree of Osaka Prefecture University.

Takeyasu, Kazuhiro and Okita, Katsuhisa (2007), Optimization in Sea and Air Freight Transport Utilizing Genetic Algorithms, Memoir of Osaka Prefecture University, 52(3), 59-72.

Amemiya, Takashi and Okita, Katsuhisa (2009), Basic Consideration for the Optimal Allocation of Three Modes Intercontinental Transportation of Seasonal Products, Journal of Business Administration and Information, Memoir of Setsunan University, 17(1).

Amemiya, Takashi and Okita Katsuhisa (2009), Basic Consideration for the Optimal Allocation of Three Modes Intercontinental Transportation of Seasonal Products, Proceedings $10^{\text {th }}$ APIEMS.

Hiraki, S., Ichimura, T., and Ishii, K. (2008), Designing of a Global Logistics System for the ICGCPS under Considering Overseas Markets, Journal of Industrial Engineering and Management Systems, 7 (3), 89-196.

Chen, H. Y. and Wu, H. C. (2009), Decentralized Supply Chain Coordination with Revenue Sharing Mechanism: Transfer Pricing Heuristics and Revenue Share Rates Journal of Industrial Engineering and Management Systems, 8(4), 213-220.

Chong, L. and Kennedy, D. Tools for Logistics Planning Journal of Industrial Engineering and Management Systems, 5(2), 57-67. 